\title{
I rimedi giurisprudenziali della Corte Europea dei diritti dell'uomo sono sufficienti per tutelare la libertà dei media?
}

\author{
Başak Çali ${ }^{1}$
}

"Aumentare la sicurezza dei giornalisti e degli altri operatori mediatici viene considerato una questione di massima urgenza. È stato ripetutamente ribadito che sussiste la necessità di abbandonare il corrente affidamento a misure reazionarie a favore di un approccio più preventivo" (McGonagle 2014).

\section{INTRODUZIONE}

La legislazione europea in materia di diritti umani ritiene che la libertà di espressione, in particolare la protezione per i giornalisti affinché possano diffondere le informazioni e per il pubblico affinché possa riceverle, sia un pilastro fondamentale della protezione europea dei diritti umani. Di conseguenza, e in conseguenza della giurisprudenza sviluppatasi a partire dagli anni $70^{2}$, vengono ripetutamente proposti dinanzi alla Corte europea dei diritti dell'uomo (la Corte) casi che riguardano la libertà mediatica. Inoltre, molte sentenze relative alla libertà di espressione di alto profilo che coinvolgono la protezione dei giornalisti, non sono ancora state eseguite e pendono dinanzi al Comitato dei Ministri. ${ }^{3}$ Bisogna quindi chiedersi se il sistema europeo dei diritti umani nel complesso è adeguatamente equipaggiato per riempire lo spazio vuoto tra il valore immanente accordato alla protezione dei giornalisti e l'effettiva attuazione di tale valore nella legislazione nazionale e nella pratica.

In questa sede, il mio obiettivo è occuparmi della protezione dei diritti dei giornalisti tramite l'utilizzo di uno degli strumenti disponibili per consolidare il diritto: i rimedi giurisprudenziali della Corte europea dei diritti

1 Direttice del Centro di diritto internazionale pubblico e professore associato di Diritto internazionale, Koç University, Istanbul, Turchia, bcali@ku.edu.tr.

2 Le statistiche della Corte europea dei diritti dell'uomo mostrano che, tra 1959 e 2014, la Corte rilevò 594 violazioni della libertà d'espressione (Corte europea dei diritti dell'uomo 2015a).

3 Vedi, per esempio, Dink c. Turchia; Incal Group c. Turchia; Muradova c. Azerbaigian. 
dell'uomo. A mio avviso, i rimedi giurisprudenziali rappresentano la giurisprudenza che cerca di porre rimedio in maniera effettiva alle violazioni dei diritti umani esistenti e di prevenire che simili violazioni si ripetano in futuro. I rimedi giurisprudenziali si distinguono dalla giurisprudenza di merito. In quest'ultima la Corte identifica le modalità chiave per interpretare i diritti, i loro obiettivi e le circostanze in cui essi possono essere legittimamente ristretti. I rimedi giurisprudenziali mirano ad un approccio implementativo nei singoli casi in questione. Trattano i problemi sottostanti che causano le violazioni dei diritti in modo più diretto, i modi per prevenire le stesse in futuro e i modi specifici in cui si può porre rimedio a una singola violazione della Convenzione europea dei diritti dell'uomo (la Convenzione).

Nel presente capitolo punto a dare una risposta a due questioni collegate tra loro. In primis, quali sono le caratteristiche centrali dei rimedi giurisprudenziali della Corte europea dei diritti dell'uomo nel campo della protezione dei giornalisti? In secundis, i rimedi giurisprudenziali della Corte sono adeguati in termini di contributi effettivi alle misure generali e individuali maggiormente necessarie nel campo della protezione dei giornalisti e per assicurare la libertà dei media?

Per dare una risposta a queste domande mi soffermerò in questa sede sul modello delle sentenze dichiarative, il quale costituisce l'approccio standard della Corte nei rimedi giurisprudenziali. Sostengo che tale modello presenti due punti deboli nell'ambito della libertà dei media.

Innanzitutto, i rimedi giurisprudenziali della Corte non inviano segnali forti agli Stati in riferimento alla loro ottemperanza ai principi della libertà giornalistica e alla protezione dei giornalisti. Ciò nonostante l'alta considerazione giurisprudenziale sostanziale sviluppata nei confronti dell'importanza della libertà di espressione in una democrazia plurale e l'accurata valutazione del margine di apprezzamento degli Stati nell'infrangere tali diritti. La chiara enunciazione di principi non porta alla piena esecuzione delle sentenze della Corte, poiché, da un lato, gli Stati tendono a limitarsi al minimo richiesto da tali sentenze e, dall'altro, il processo di esecuzione delle sentenze dinanzi al Comitato dei ministri è particolarmente lento e convenzionale.

In secondo luogo, l'approccio della Corte europea dei diritti dell'uomo, la quale si focalizza sulla creazione di standard invece di prevenire violazioni in futuro, porta alla reiterazione di casi da parte di Paesi in cui l'attuazione dei giudicati in materia di diritti umani non viene considerata prioritaria.

Per riassumere, è necessario che la Corte europea dei diritti dell'uomo adotti un approccio più strategico e preventivo al fine di facilitare la restri- 
zione dello spazio tra i principi della libertà mediatica e la loro attuazione alla base. Nonostante il Comitato dei Ministri sia confrontato con l'esecuzione delle sentenze in materia di diritti umani e l'adeguatezza dei rimedi posti in essere, ${ }^{4}$ la protezione dei giornalisti è un'area in cui la Corte può fare leva sul proprio "potere normativo" 5 nei confronti degli Stati in modo più effettivo al fine di sostenere il lavoro del Comitato dei Ministri.

Nelle parti che seguono, comincerò dal rapporto tra i rimedi giurisprudenziali e l'effettiva protezione dei diritti in termini generali ed esporrò le caratteristiche centrali dei rimedi giurisprudenziali del sistema europea dei diritti umani. Esplorerò poi le tendenze generali nei rimedi giurisprudenziali della Corte nel campo della libertà di espressione, focalizzandomi in particolare sulla protezione dei giornalisti e delle libertà mediatiche. In terzo luogo, parlerò della debolezza dei rimedi giurisprudenziali attuali per l'effettivo ancoraggio dei diritti, difenderò un approccio più strategico da parte della Corte nei confronti della libertà dei media e risponderò alle potenziali obiezioni per costruire un ruolo chiaramente delineato, ma più attivo per la Corte in tale campo. Concluderò suggerendo strade da percorrere all'interno del quadro giuridico del sistema corrente per chiudere lo spazio tra i principi e la pratica nel campo della protezione dei diritti dei giornalisti e della libertà dei media all'interno dell'ambito di azione del Consiglio d'Europa.

\section{I RIMEDI GIURISPRUDENZIALI DEL SISTEMA EUROPEO DEI DIRITTI UMANI}

Il sistema europeo dei diritti umani è noto in particolare per la giurisprudenza sostanziale della Corte europea dei diritti dell'uomo. Con le sue oltre 12.000 sentenze, la Corte ha avuto l'opportunità di interpretare tutte le previsioni sostanziali della Convenzione europea dei diritti dell'uomo sin

4 A fianco delle principali attribuzioni relativi all'esecuzione, il Comitato dei Ministri si è incaricato dell'importante compito di definire gli standard relativi alla protezione dei giornalisti (vedi Comitato dei Ministri del Consiglio d'Europa 2014). La Federazione russa introdusse una riserva relativa a questa dichiarazione del Comitato dei Ministri; nello specifico negò la sua applicazione ad "altri attori mediatici”, poichè considerò questo termine non specifico e non presente in documenti vincolanti a livello internazionale.

5 Il potere normativo della Corte consiste nel potere guida nei confronti degli Stati membri del Consiglio d'Europa in relazione alle misure appropriate da adottare nel campo dei diritti umani. Per ulteriori approfondimenti, vedi Çalı e Koch (2014). 
da quando ha giudicato il proprio primo caso nel 1960. Una tale interpretazione ha portato a un lavoro giurisprudenziale rivoluzionario nel definire l'ambito dei diritti e i motivi inammissibili di violazione degli stessi considerando la Convenzione uno strumento vivente. ${ }^{6}$ La Corte dispone di una casistica ben sviluppata e ha elaborato modi di valutazione specifici per interpretare le singole previsioni della Convenzione. Esse stabiliscono come gli obblighi positivi vadano concepiti per ogni categoria di diritti, ${ }^{7}$ come i diritti qualificati possano essere attuati in circostanze concrete e quale relazione esista tra il margine di apprezzamento delle autorità statali e il rispetto dei diritti. ${ }^{8}$ Nella sfera degli obblighi positivi, la Corte ha fatto significanti progressi nello sviluppo di obblighi procedurali collegati agli obblighi positivi sostanziali che scaturiscono dalle previsioni della Convenzione. $^{9}$

Mentre la Corte ha sviluppato un'importante serie di sentenze riguardanti i diritti nel corso del tempo, ha mantenuto la propria impostazione conservatrice nello sviluppo di rimedi giurisprudenziali che corrispondano alle violazioni della Convenzione. I rimedi giurisprudenziali della Corte sono rimasti, nella gran parte della loro esistenza, all'interno del modello delle sentenze dichiarative. La Corte spiega, nelle proprie sentenze, i motivi per i quali uno Stato ha violato la Convenzione, ma non offre una guida specifica su (I) come porre rimedio alla violazione in tute le sue dimensioni o (II) quali misure debba intraprendere uno Stato al fine di prevenire nuove violazioni in futuro. Al contrario, la Corte assegna compensazioni pecuniarie o non alle vittime di violazioni dei diritti umani ex art. 41 della Convenzione e sulla base dell'equità. ${ }^{10} \mathrm{La}$ Corte ha giustificato ciò indican-

6 Vedi, a titolo di esempio rappresentativo, Demir e Baykara c. Turchia, 12 novembre 2008 [GC], paragrafi 65-68.

7 Sullo sviluppo degli obblighi, vedi Mowbray (2004).

8 Vedi, a titolo di esempio rappresentativo, Smith e Grady c. Regno Unito.

9 Per quanto riguarda le violazioni dell'articolo 2 e dell'articolo 3, questa fonte è particolarmente dettagliata.

Vedi in generale, Sayev ed altri c. Russia, Ricorso n. 43368/04, 21 giugno 2011, paragrafi 186-187; Anguelova c. Bulgaria, Ricorso n. 38361/97, 13 giugno 2002, paragrafo 161; Mahmut Kaya c. Turchia, Ricorso n. 22535/93, Sentenza del 28 marzo 2000, paragrafo 107; e, per quanto concerne le accuse di maltrattamento, vedi, per esempio, El-Masri c. Ex Repubblica Jugoslava di Macedonia, paragrafo 255; Labita c. Italia, Ricorso n. 26772/95, 6 aprile 2000, paragrafo 131. Vedi anche Brems e Lavrysen (2013).

10 È stato evidenziato dagli autori che la Corte ha seguito un'interpretazione stretta della nozione di "equa riparazione" contenuta nell'Articolo 41, interpretando "equa riparazione" solamente nel suo significato di compensazione. Vedi Shelton (2006) p.197. 
do la suddivisone del lavoro tra il Comitato dei Ministri e la Corte europea dei diritti dell'uomo nell'esecuzione delle sentenze $e x$ art. 46 della Convenzione. ${ }^{11}$ Tradizionalmente, ha ritenuto che essa non goda del potere di porre in essere grandi misure correttive. ${ }^{12}$

È ben risaputo che, a partire dal 1990, la Corte si sia scontrata con un numero di casi impressionante. La causa di ciò, un aumento dei casi provenienti da un numero ristretto di Stati membri ${ }^{13}$ del Consiglio d'Europa e casi ripetuti, ${ }^{14}$ ha evidenziato una carenza di attuazione effettiva degli standard previsti dalla Convenzione negli ordinamenti nazionali, nella policy e nella casistica giurisprudenziale. Il sistema della Convenzione nel suo complesso ha risposto a questo elevato numero di casi adottando una serie di misure. A livello istituzionale vi furono tentativi di aumentare l'efficienza del lavoro della Corte. ${ }^{15}$ A livello politico il Consiglio d'Europa rese l'effettiva implementazione delle sentenze riguardanti i diritti umani un tema di policy centrale. Questo interesse è riscontrabile nelle dichiarazioni incentrate sull'implementazione da parte del Comitato dei Ministri. Circa nel 2000, il Comitato dei Ministri ha suggerito che ai casi in cui la Corte ha rilevato una violazione andasse meglio posto rimedio consentendo nuovi processi (Comitato dei Ministri del Consiglio d'Europa 2000). In particolare, nel 2004, il Comitato dei Ministri ha richiesto l'assistenza della Corte per mezzo di una maggiore guida sull'implementazione delle sue sentenze (Comitato dei Ministri del Consiglio d'Europa 2004). Il Comitato dei Ministri ha inoltre indicato le falle istituzionali nazionali nell'esecuzione chiedendo agli Stati di creare punti centrali per l'esecuzione (Comitato dei

11 La nota posizione della Corte sul tema è la seguente: "La Corte ribadisce che le sue sentenze sono essenzialmente di natura dichiarativa e che, in generale, spetta principalmente allo Stato interessato scegliere, sotto il controllo del Comitato dei Ministri, i mezzi da utilizzare all'interno del proprio ordinamento giuridico interno per adempiere all'obbligo di cui all'Articolo 46 della Convenzione, a condizione che tali mezzi siano compatibili con le conclusioni esposte nella sentenza della Corte ". Vedi, tra gli altri, Scozzari e Giunta c. Italia [GC], n. 39221/98 e 41963/98, paragrafi 249, ECHR 2000-VIII; e Brumărescu c. Romania (equa riparazione) [GC], n. 28342/95, paragrafo 20, ECHR 2001-I.

12 Cf. Saïdi c. Francia, 20 settembre 1993, paragrafo 47, Serie A n. 261-C.

13 Nel maggio 2015, i Paesi - Ucraina, Russia, Turchia, Italia e Romania - costituivano il $68,5 \%$ di tutti i casi in sospeso dinanzi alla Corte, ovvero 64.350 casi. Vedi Corte europea dei diritti dell'uomo (2015b).

14 A luglio 201439.721 degli 8.5000 casi iscritti al ruolo della Corte erano ricorsi ripetitive. Vedi Corte europea dei diritti dell'uomo (2014).

15 Informazioni relative al processo di rinnovamento della Corte europea dei diritti dell'uomo sono accessibili al link: www.echr.coe.int/Pages/home.aspx?p=court/ref orm\&c=\#n13740528735758554841286_pointer, ultimo accesso: 21 luglio 2015. 
Ministri del Consiglio d'Europa 2008). L'esecuzione delle sentenze della Corte in forma di misure generali e specifiche ha continuato a contraddistinguere il lavoro del Comitato dei Ministri per mezzo di dichiarazioni di alto livello a Interlaken, Brighton, Izimir e, più recentemente, a Bruxelles nel 2015. ${ }^{16}$ Anche l'Assemblea parlamentare del Consiglio d'Europa sostiene tutto ciò, richiedendo alla Corte di fornire più frequentemente guide sulle misure generali e individuali (Assemblea parlamentare del Consiglio d'Europa 2000: paragrafo 11 B).

Oltre alla tematica dell'esecuzione delle sentenze della Corte, i processi politici all'interno del Consiglio d'Europa hanno inoltre evidenziato la necessità che la Corti presti sufficiente attenzione agli sforzi compiuti in buona fede dagli ordinamenti nazionali per conferire efficacia ai diritti della Convenzione all'interno dei relativi sistemi giuridici. Nella Dichiarazione di Brighton ciò è culminato nell'enfatizzazione del principio di sussidiarietà, ritenendo che gli ordinamenti nazionali siano il primo posto in cui rispettare i diritti sanciti dalla Convenzione. Il Protocollo n. 15 riprende tale tematica, in cui il "margine di apprezzamento" è stato aggiunto al Preambolo della Convenzione.

Questi due sviluppi gemelli, il focus rivolto all'implementazione e l'attenzione nei confronti della sussidiarietà del sistema della Convenzione, hanno inoltre creato spostamenti qualitativi nell'interpretazione della Convenzione da parte della Corte. Vi sono due caratteristiche corrispondenti dello spostamento in questione.

In primis, la Corte ha risposto in modo positivo all'invito a rendere le proprie sentenze più "agevoli da attuare" specificando i tipi di misure che vanno realizzate da parte degli ordinamenti giuridici nazionali o nei dettami delle sentenze della Corte oppure aggiungendo osservazioni finalizzate all'esecuzione nelle parti delle sentenze della Corte ex art. 46 o tramite una procedura introdotta nel 2004, le c.d. "sentenze pilota".

Per quanto riguarda la casistica sostanziale, la Corte ha iniziato a indicare, nelle massime delle proprie sentenze, che la presenza e/o l'assenza di un contesto legislativo o normativo può portare a violazioni dei diritti umani. ${ }^{17}$ In questo modo, la Corte ha scelto di segnalare agli Stati e al Comitato dei Ministri quali tipi di misure legislative, cambi di mentalità e addirittura misure individuali urgenti sono richieste al fine di prevenire violazio-

16 Vedi "Dichiarazione di Brighton", paragrafi 3 and 4; anche paragrafo 12.c. "Dichirazione di Interlaken", paragrafo 3, e Piano d'azione, paragrafo E.9; e "Dichiarazione di Izmir”, paragrafo 6 (Comitato dei Ministri del Consiglio d'Europa 2010, 2011, 2012).

17 Vedi, inter alia, Opuzc. Turchia. 
ni di diritti umani in casi simili in futuro e a porre rimedio alle questioni poste alla sua attenzione.

La Corte ha iniziato, in particolare a partire dal 2004, a fare un uso innovativo dell'art. 46 della Convenzione europea dei diritti dell'uomo creando una parte separata nelle proprie sentenze in cui vengono affrontate le misure individuali e generali. Ha fatto uso dell'art. 46 per illustrare la necessità di adottare misure specifiche individuali, tuttavia l'esatta specificazione delle misure individuali è stata, finora, rara. ${ }^{18} \mathrm{La}$ Corte ha, per esempio, chiesto di porre termine alla detenzione illegittima ed alla carcerazione illegittima, la rimozione degli ordini di detenzione, l'esecuzione di una particolare sentenza della Corte e di fornire informazioni ad un richiedente da parte dei servizi segreti. ${ }^{19}$ Nei casi in cui la Corte specifica tali misure, partendo dal proprio modello declaratorio, ciò è giustificato da un prova di "necessità logica”. Qui la Corte statuisce che nessun'altra misura soddisferebbe, tramite di una conclusione logica, il rimedio richiesto. ${ }^{20}$ Per quanto riguarda le misure generali, la Corte fa riferimento all'art. 46 il quale dispone che una violazione in un caso particolare è sistematica o ha dei connotati sistematici. La discussione separata di problemi sistematici o strutturali a cui vanno posti rimedi per prevenire violazioni future o per ottemperare ai giudicati è spesso ritenuta un segnale più forte nei confronti degli Stati e del Comitato dei Ministri rispetto a qualsiasi discussione di problematiche simili nelle massime della Corte.

La risposta finale della Corte alla crisi attuativa è stata data istituendo la "procedura delle sentenze pilota" (Regolamento della Corte, Corte europea dei diritti dell'uomo, 2011). La procedura delle sentenze pilota è la più importante per il problema dei casi ripetuti. Proclamando un caso pilota, spesso uno tra centinaia di casi simili, la Corte identifica problemi sistematici e strutturali che conducono alle ripetute violazioni dei diritti umani. Richiede che gli Stati contrastino i problemi identificati con urgenza, spes-

18 Per esempi rilevanti, vedi Assidanze c. Georgia, Volkov c. Ucraina, Fattulayev c. Azerbaigian.

19 Vedi, inter alia, Assanidze c. Georgia, Fattullayev c. Azerbaigian, Emre c. Svizzera, Karanoniç c. Bosnia- Erzegovina.

20 Come afferma la Corte: "In alcuni casi, la natura della violazione rilevata può essere tale da non lasciare una scelta reale sulle misure correttive richieste, portando la Corte a decidere per un'unica di queste misure correttive." Assanidze c. Georgia [GC], n.71503/01, paragrafo 202. In questo contesto, l'uso di misure individuali attuate da parte della Corte è qualitativamente divergente dall'uso di queste stesse misure da parte della Corte interamericana dei diritti umani. Quest'ultima stabilisce una lista completa di misure individuali che necessitano di essere adottate in ogni singolo caso. Vedi Shelton (2000). 
so tramite l'introduzione di leggi, all'interno di un arco di tempo specificato. ${ }^{21} \mathrm{Nel}$ frattempo, la Corte congela il trattamento di casi simili e attende informazioni dal Comitato dei Ministri per l'esecuzione delle sentenze pilota.

In secundis, e come risposta alla richiesta di un maggiore impegno diretto con il principio di sussidiarietà, la Corte ha introdotto due nuove teorie, la "teoria della responsabilità dellle corti" e la "teoria deilla responsabilità dei parlamenti". ${ }^{22}$ Con la prima delle due, la Corte riconosce che, a condizione che i tribunali nazionali stiano applicando i principi consolidati della Convenzione nelle loro sentenze, rinuncia a rivedere i risultati sostanziali delle deliberazioni. ${ }^{23}$ Secondo la formulazione della Corte, richiederebbe "forti ragioni" per giudicare direttamente gli esiti delle decisioni. ${ }^{24}$ Nella teoria della responsabilità dei parlamenti, la Corte riconosce che porrà adeguata attenzione alle seguenti questioni: (I) se le deliberazioni politiche sostanziali ponderano con cautela i diritti in gioco, e (II) se la qualità procedurale delle deliberazioni è sufficientemente inclusiva in modo tale da tenere conto degli interessi di tutti gli stakeholder rilevanti. ${ }^{25}$ L'impatto di tali due dottrine, in fase di evoluzione, incentrate sulla nozione di sussidiarietà per i rimedi giurisprudenziali della Corte, consiste nella valutazione esplicita dei rimedi giurisprudenziali e legislativi disponibili negli ordinamenti nazionali alla fonte. In altre parole, la Corte ha assunto un approccio più esplicito nel valutare l'adeguatezza dei rimedi nazionali in forma di decisioni giudiziarie e deliberazioni parlamentari. Ha aperto un dialogo con le proprie controparti giudiziarie sulla corretta applicazione dei principi previsti dalla Convenzione.

\section{I RIMEDI GIURISPRUDENZIALI DEL SISTEMA EUROPEO DEI DIRITTI UMANI E LA LIBERTÀ DEI MEDIA}

Vorrei quindi valutare l'impatto del mutamento qualitativo nell'interpretazione della Convenzione verso un approccio più focalizzato sull'aspetto at-

21 La Corte propone un tempo limite di un anno o diciotto mesi, ma non ci sono regole chiare sui limiti di tempo adeguati e questi limiti devono essere stabiliti caso per caso (Corte europea dei diritti dell'uomo 2015d).

22 In relazione ai contenuti di questa dottrina, vedi Çalı (2013).

23 Per un esempio di applicazione di questa dottrina, vedi Van Hannover c. Germania (2).

24 Ibid., paragrafo 107.

25 Animal Defenders c. Regno Unito, SAS c. Francia. 
tuativo nel campo della libertà dei media. In che modo lo sviluppo dei rimedi giurisprudenziali da parte della Corte nella propria casistica ha, in generale, contribuito ad assicurare rimedi più effettivi nei casi in cui le violazioni della libertà dei media hanno luogo? Così facendo, miro ad analizzare le massime della casistica della Corte per quanto esse possano creare standard ed essere improntate sull'attuazione, così come l'uso di misure specifiche individuali, ricorrendo alle sentenze riferite all'art. 46 e alle sentenze pilota.

\subsection{Le sentenze che creano standard della Corte e la loro esecuzione}

È ampiamente risaputo che la Corte considera la libertà di espressione una pietra miliare della Convenzione. ${ }^{26}$ Secondo la casistica della Corte, la libertà di espressione rappresenta un indicatore centrale della governance democratica. Tale diritto non va solo protetto in quanto tale: la sua protezione è strumentale per la salute di un regime plurale democratico. ${ }^{27}$ In questo contesto, la libertà dei media, in particolare la protezione dei giornalisti ${ }^{28}$ e degli organi di stampa, ${ }^{29}$ e, più recentemente, di cittadini e di organi non governativi che forniscono informazioni per il pubblico dibattito, ${ }^{30}$ godono di speciali protezioni ai sensi dell'art. 10 della Convenzione. Gli operatori mediatici sono "pubblici guardiani". ${ }^{31}$ La Corte evidenzia nella propria giurisprudenza sostanziale riguardante la libertà di stampa che $\mathrm{i}$

26 Stoll c. Svizzera, n. 69698/01, 10 dicembre 2007, paragrafo 110.

27 MGN c. Regno Unito, Applicazione n. 39401/04, Sentenza del 18 gennaio 2001 al paragrafo 141; Corte europea dei diritti dell'uomo, Flux c. Moldavia, Applicazione n. 28702/03, Sentenza del 12 novembre 2007 al paragrafo 43; Corte europea dei diritti dell'uomo, Castells c. Spagna, Ricorso n. 11798/85, Sentenza del 23 aprile 1992 al paragrafo 43; Corte europea dei diritti dell'uomo, Thorgeir Thorgeirson $c$. Islanda, Ricorso n. 13778/88, Sentenza del 25 giugno 1992 al paragrafo 63.

28 Cfr. Observer e Guardian c. Regno Unito, 26 novembre 1991, Serie A n. 216; De Haes e Gijsels c. Belgio, 24 febbraio 1997, paragrafo 37, Recueil 1997-I.

29 Cfr. il caso Centro Europa 7 S.r.l. e Di Stefano c. Italia; RTBF c. Belgio, Sentenza del 29 marzo 2011.

30 Corte europea dei diritti dell'uomo, Társaság a Szabadságjogokért c. Ugheria, Ricorso n. 37374/05, Sentenza del 14 aprile 2009; Corte europea dei diritti dell'uomo, Youth Initiative for Human Rights c. Serbia, Ricorso n. 48105/08, Sentenza della Grande Camera del 22 aprile

2013 al paragrafo 20.

31 Observer e Guardian c. Regno Unito, 26 novembre 1991, paragrafo 59, Serie A n. 216, e The Sunday Times c. Regno Unito (n. 2), 26 novembre 1991, paragrafo 50, Serie A n. 217. 
giornalisti godono di protezione sia in quanto individui sia in quanto giornalisti. ${ }^{32}$ La ragione di ciò consiste nell'importanza della protezione dei giornalisti e dei loro mezzi per fornire informazioni pubbliche alla società nel suo complesso. Una carenza di protezione per i giornalisti lede sia i diritti dei giornalisti che il diritto del pubblico di ricevere informazioni. Secondo la celebre formulazione della Corte, qualsiasi tipo di riduzione dei diritti dei giornalisti e dei media produce un "effetto dissuasivo", 33 affievolendo sia i diritti individuali che la qualità di una società democratica e plurale. Le restrizioni all'attività dei media vanno interpretate in maniera molto severa, lasciando agli Stati un margine di apprezzamento molto ristretto e giustificando l'intensità di revisioni da parte della Corte. ${ }^{34}$ Uno Stato deve accettare un ampio ambito di obblighi positivi al fine di proteggere i giornalisti, anche da parte di terzi, ${ }^{35} \mathrm{e}$ al fine di creare un ambiente favorevole in cui i giornalisti siano in grado di svolgere il loro lavoro. ${ }^{36}$

Nonostante l'importanza centrale della libertà dei media per la Convenzione nel suo complesso, gli standard in riferimento alla protezione della libertà dei media sono stati sviluppati secondo il modello delle sentenze dichiarative. Ciò significa che in ogni caso in cui la Corte si è occupata della libertà dei media, essa si è focalizzata sull'aspetto giurisprudenziale sostanziale del caso. Ha comunque lasciato l'ambito di implementazione effettiva della sentenza del singolo Stato sotto la supervisione del Comitato dei Ministri. Così facendo, la Corte ha evidenziato il limitato ambito di margine di apprezzamento, la necessità di leggi accessibili ${ }^{37}$ e la necessità ${ }^{38}$ e proporzionalità ${ }^{39}$ delle misure adottate dalle autorità nazionali. Indubbiamente le decisioni della Corte hanno contribuito in maniera significante alla discussione sui rimedi generali e individuali adeguati richiesti dagli ordinamenti nazionali dinanzi al Comitato dei Ministri. ${ }^{40}$ Ciò è, in ogni caso, ri-

32 Matúzc. Ungheria (2014).

33 Cumpana e Mazare c. Romania, 33348/96, Sentenza (merito ed equa riparazione), Corte (Grande Camera), 17 dicembre2004.

34 Hertel c. Svizzera, 25 agosto 1998, paragrafo 46, Reports of Judgments and Decisions 1998-VI; Tammer c. Estonia, n. 41205/98, paragrafo 59, ECHR 2001-I; e Steel e Morris c. Regno Unito, n. 68416/01, paragrafo 87, ECHR 2005.

35 Özgür Gündem c. Turchia, n. 23144/93, 16 marzo 2000, paragrafi 43 e 46.

36 Dink c. Turchia, n. 2668/07, 6102/08, 30079/08, 7072/09 e 7124/09, 14 settembre 2010, paragrafo 137.

37 Editorial Board of Pravoya dela e Shtekel c. Ucraina (2011), paragrafo 65.

38 Sürek c. Turchia (n. 1) [GC], n. 26682/95, paragrafo 61, ECHR 1999-IV.

39 Erla Hlynsdottir c. Islanda (n.3) (2015).

40 Per una visione d'insieme sugli standard che emergono dalla casistica della Corte relativa alla libertà dei media e alla protezione dei giornalisti, vedi Leach (2013). 
masto una tematica implicita all'interno della giurisprudenza della Corte, senza possedere le qualità di rimedio giurisprudenziale specifico focalizzato sulla prevenzione o su un approccio comprensivo volto al rimedio per $\mathrm{i}$ singoli danneggiati al di là di un ristoro economico dal valore simbolico.

\subsection{Gli standard di Strasburgo: fulcro sul ragionamento delle corti nazionali}

Si può sostenere che, analizzando la casistica consolidata della Corte, uno Stato che sia parte in giudizio possa ottenere una guida sufficiente su quali standard esso debba cercare di raggiungere e sul modo in cui esso debba porre rimedio alle violazioni dei diritti individuali. Infatti, le sentenze della Corte sulla libertà dei media indicano spesso, se lette in maniera accurata, quali errori futuri potrebbero portare a future sentenze che accertino ulteriori violazioni. Nella giurisprudenza preminente della Corte sulla libertà giornalistica, sarebbe, tuttavia, ragionevole sostenere che la Corte di Strasburgo si è maggiormente preoccupata di offrire indicazioni specifiche alle corti nazionali più che ad altri partner per l'implementazione nei contesti nazionali - ovvero i parlamenti, in governi e le agenzie regolatrici. La Corte punta a inviare messaggi chiari alle corti nazionali in merito alla necessità di adottare una prospettiva incentrata sulla libertà di espressione e sull'esigenza di valutare con cautela la necessità e la proporzionalità dell'interferenza nella libertà di parola.

Si prenda come esempio della preoccupazione principale della Corte di porre standard per le proprie controparti nazionali la discussione sulle lacune argomentative delle corti nazionali ungheresi nel caso Matúz. Qui la Corte di Strasburgo ha deciso che i giornalisti che lavorano per un'emittente pubblica continuano a godere della libertà di espressione in quanto giornalisti e non possono essere trattati alla stregua di semplici dipendenti pubblici:

per quanto riguarda il modo in cui è stata esaminata la causa lavorativa dell'appellante, le corti nazionali hanno ritenuto che il mero fatto che l'appellante abbia pubblicato il libro sia sufficiente per concludere che egli abbia agito a danno del proprio datore di lavoro. In ogni caso, esse non hanno prestato attenzione all'argomentazione dell'appellante, il quale sosteneva che egli stava facendo esercizio della propria libertà di espressione nel pubblico interesse, limitando la loro analisi nel ritenere che abbia violato i propri obblighi contrattuali. Inoltre, la sentenza della Corte suprema ha ritenuto che la questione si limitasse a un contenzioso di natura lavorativa e non riguardasse i diritti fondamentali 
dell'appellante. Di conseguenza, le Corti nazionali non hanno esaminato se e come la materia oggetto della pubblicazione dell'appellante e il contenuto della stessa possano aver riguardato l'ambito ammissibile di restrizione della libertà di espressione, benché un tale approccio sarebbe stato, in linea di principio, compatibile con gli standard posti dalla Convenzione. ${ }^{41}$

Tale sentenza offre ampia guida per le corti nazionali che si occuperanno di casi futuri simili, evidenziando che, nei casi in cui sono coinvolti giornalisti, le corti nazionali devono tenere in considerazione la protezione della libertà dei giornalisti in modo prioritario. Quando la natura delle sentenze riguardanti la libertà giornalistica costituisce in effetti un dialogo giurisprudenziale tra la Corte e le proprie controparti nazionali e la Corte fa da guida, per mezzo delle proprie sentenze, alle corti nazionali per l'applicazione degli standard della Convenzione, il modello di sentenza dichiarativa, con ampio spazio dedicato agli standard sostanziali, risulta particolarmente indicato. In questo contesto, essendo sia la Corte di Strasburgo sia le Corti nazionali istituzioni giudiziarie indipendenti, il modo migliore per proteggere la libertà dei giornalisti tramite le corti avverrebbe tramite la creazione di standard da parte della Corte europea dei diritti dell'uomo. La Corte ha altresì confermato ciò nelle proprie sentenze. Per esempio, nel caso Animal Defenders, la Corte ha esplicitamente statuito che "la qualità di ... una revisione giudiziaria della necessità della misura è di particolare importanza in questo contesto, inclusa l'applicazione di un relativo margine di apprezzamento". ${ }^{42}$ La Corte si aspetta che violazioni analoghe verranno evitate tramite la traduzione e l'effettiva divulgazione di tale sentenza, uno standard richiesto dal Comitato dei Ministri agli Stati membri, e tramite la chiara comunicazione degli standard da parte di Strasburgo alle corti nazionali.

Accertato il dialogo sviluppato dalla Corte con le corti nazionali a riguardo delle libertà giornalistiche, è altresì importante notare che, nonostante la specificità delle massime, tale approccio riscontra comunque difficoltà nell'assicurare l'effettiva attuazione delle sentenze, come facilmente riscontrabile sulla base dell'approccio della Corte nei confronti delle normative generali in materia di diffamazione. I processi per il reato di diffamazione sono divenuti quale forma di interferenza nei diritti dei giornalisti una prassi comune in alcuni Paesi membri del Consiglio d'Europa. A

41 Matúz c. Ungheria, paragrafo 49.

42 Animal Defenders International c. Regno Unito [GC], N. 48876/08, paragrafo 108, CEDU 2013. 
partire dalla prima sentenza emessa dalla Corte sulla calunnia nel caso Lingens $c$. Austria, ${ }^{43}$ essa ha indicato, trattandosi di una questione di principi generali, che le leggi penali in materia di diffamazione non andrebbero applicate quando esistono alternative civilistiche. ${ }^{44} \mathrm{Ha}$ aggiunto che, in generale, la legislazione penale andrebbe adoperata soltanto in risposta a casi estremi di diffamazione. ${ }^{45} \mathrm{Ha}$ altresì evidenziato che, in ogni caso, l'esistenza di normative penali in materia di diffamazione non viola ex se la libertà di espressione. ${ }^{46} \mathrm{Dal}$ punto di vista della giurisprudenza sostanziale, non dichiarare le leggi penali in materia di diffamazione contrarie alla libertà mediatica incompatibili con la libertà di espressione non è, di per sé, problematico. Ciò perché la Corte applica un criterio di valutazione molto severo per il possibile uso proporzionato di tali leggi e ritiene tali leggi illegittime qualora esse vengano utilizzate nonostante esistano alternative civilistiche.

Dal punto di vista di un'implementazione effettiva, ciò comporta, in ogni modo, alcune difficoltà. Le leggi penali in materia di diffamazione possono rispondere a obiettivi molto ampi e vengono spesso utilizzate come strumento per punire in via indiretta attività giornalistiche. Anche nei casi in cui i giornalisti vengono prosciolti, i processi penali producono un effetto dissuasivo sulla libertà mediatica nel complesso. Quando esistono leggi penali in materia di diffamazione, l'approccio sostanziale della giurisprudenza della Corte fa affidamento alla cooperazione positiva dei due procuratori e dei giudici al fine di assicurare un'applicazione adeguata e moderata di tali norme. Quando gli Stati non cooperano in modo positivo o quando esistono problemi sistematici di amministrazione giudiziaria in un determinato Paese, i principi sostanziali non sono, in ogni modo, in grado di porre fine a una serie di casi che si ripetono. ${ }^{47}$ In altre parole, l'approccio che prevede la creazione di uno standard per l'uso parsimonioso del reato di diffamazione non garantisce un risultato effettivo nei Paesi in

43 Lingens c. Austria, 8 luglio 1986, Ricorso n. 9815/82.

44 Mahmudov e Agadze c. Azerbaigian, Ricorso n. 35877/04, 18 dicembre 2008.

45 Gavrilovici c. Moldavia, Ricorso n. 25464/05, 15 dicembre 2009, paragrafo 60.

46 Makarenko c. Russia, 22 dicembre 2009, Ricorso n. 5962/03, paragrafo 156.

47 Vedi, per esempio, i casi relativi al rispetto della libertà di stampa che sono oggetto di una procedura prolungata dinanzi al Comitato dei Ministri, Muradova c. Azerbaigian, Najafl c. Azerbaigian, e Rizvanov c. Azerbaigian, disponibile al link: www.coe.int/t/dghl/monitoring/execution/reports/pendingcases_EN.asp?CaseTitl eOrNumber $=\&$ StateCode $=$ AZE $\&$ SectionCode $=$ ENHANCED+SUPERVISION, consultato il 22 luglio 2015. 
cui tali normative possono essere usate per sopprimere i giornalisti a causa di ampi problemi di sistema nelle prassi processuali.

\subsection{Le decisioni normative della Corte di Strasburgo ed altri soggetti coinvolti nell'attuazione della Convenzione}

Mentre abbiamo stabilito che le sentenze improntate sulla creazione di standard offrono un'ampia guida ai giudici nazionali in merito all'utilizzo degli standard della Convenzione, la medesima analisi non vale per i legislatori, i procuratori, i governi ed altri agenti regolatori nazionali che potrebbero riguardare la garanzia della libertà mediatica. Al contrario, possiamo ritenere che la Corte fa in primis affidamento agli obblighi di buona fede degli altri soggetti coinvolti nell'attuazione, confidando che essi prestino la dovuta attenzione alle statuizioni delle sentenze di Strasburgo.

Se guardiamo alla ricerca politologica sul rispetto delle sentenze della Corte, l'opinione predominante ritiene che la pura creazione di standard spesso non comporti necessariamente un'effettiva attuazione dei giudicati. In mancanza di una guida chiara o specifica, gli Stati potrebbero scegliere una soluzione che comporti la minima anziché la massima attuazione dei giudicati (Agostinou 2013; Hillbrecht 2014). Potrebbero, inoltre, non disporre delle risorse necessarie per identificare i passi concreti per adempiere ai giudicati in tempo ragionevole (Chayes and Chayes 1998). Nel sistema europeo dei diritti umani, il Comitato dei Ministri monitora la specificazione dei rimedi, e il Segretariato per l'esecuzione delle sentenze della Corte europea dei diritti dell'uomo offre l'assistenza tecnica necessaria per l'esecuzione dei giudicati. ${ }^{48}$ In altre parole, le sentenze di Strasburgo non vengono semplicemente lasciate al loro destino.

Il meccanismo secondario incontra, tuttavia, importanti ritardi nel monitoraggio e sussiste il rischio di sottomonitoraggio e di monitoraggio politicizzato. ${ }^{49}$ Il fulcro della Corte in relazione allo sviluppo di standard sostanziali più che ad un effettivo rimedio giurisprudenziale potrebbe, di conseguenze, non essere produttivo, ovvero non sfociare in un'effettiva attuazione dei giudicati da parte degli organi politici.

Sorge, sicuramente, un dilemma ovvio. La Corte rischia o di impegnarsi troppo nell'esecuzione oppure di offrire una cornice implementative inef-

48 Su questo sistema di conformità, vedi Çalı e Koch (2014).

49 Ibid. 
ficace per i diritti di un gruppo protetto percepito come la pietra miliare di una democrazia plurale.

Tale dilemma lo si può vedere nei casi di obblighi positivi che riguardano le libertà giornalistiche, nei quali la Corte richiede che gli Stati introducano nuove leggi al fine di offrire protezione alle medesime libertà. Due casi turchi ne sono un ottimo esempio. Seguendo il proprio approccio dichiarativo, la Corte ha indicato nel dispositivo di Özgür Gündem che lo Stato avesse l'obbligo positivo di proteggere $\mathrm{i}$ giornalisti da terze controparti ostili. ${ }^{50}$ Nella sentenza Dink la Corte si è spinta oltre e ha indicato che gli Stati hanno l'obbligo di creare un "sistema efficace per proteggere autori e giornalisti" e "un ambiente che consenta la piena partecipazione a dibattiti aperti". ${ }^{51}$ Portati alla loro piena conclusione, tali obblighi richiedono rimedi preventivi espliciti e meccanismi di allerta precoci in caso di attacchi alla sicurezza dei giornalisti, così come leggi sulla stampa che non consentano la nascita di monopoli.

Se osserviamo la gamma di misure generali messe in campo dalla Turchia a partire dal 2007, constatiamo che esse includono: la traduzione in turco della sentenza e la sua divulgazione, attività di formazione di consapevolezza per giudici e pubblici ministeri, una circolare del Ministero dell'Interno del 17 settembre 2010 sulle misure protettive per "persone minacciate" e un emendamento all'art. 31 del Codice penale, ai sensi della cui previsione i pubblici ministeri necessitano dell'autorizzazione del Ministero della Giustizia, che ha pertanto portato ad indagare su un numero minore di individui. ${ }^{52}$ Mentre il caso viene tuttora supervisionato, si può, sulla base delle misure adottate fino a questo momento, rilevare una discrepanza tra lo spirito del dispositivo e le misure adottate. Mentre la sentenza fa riferimento ad un sistema efficace e preventivo per proteggere i giornalisti, le misure generali si riferiscono ad una circolare per tutte le persone minacciate, affievolendo, in tal modo, l'idea che le misure generali debbano essere incentrate sui giornalisti vulnerabili. Inoltre, le misure non indicano alcuno sforzo da parte delle autorità di mettere in campo una valutazione dell'ambiente mediatico per stabilire se si tratti di un ambiente fruibile da parte di tutti in termini di accesso e di libera compartecipazione. Di

50 Özgür Gündem c. Turchia.

51 Dink c. Turchia, n. 2668/07, 6102/08, 30079/08, 7072/09 e 7124/09, 14 settembre 2010, paragrafo 137.

52 Vedi Dink c. Turchia, (procedura prolungata) disponibile al link: www.coe.int/t/dg hl/monitoring/execution/Reports/pendingCases_en.asp?CaseTitleOrNumber=Din $\mathrm{k} \&$ StateCode $=\&$ Section Code $=$, accesso: 22 luglio 2015 . 
conseguenza, le misure generali confermano la tesi di coloro che considerano gli Stati degli esecutori minimalistici.

\subsection{Le sentenze della Corte incentrate sull'esecuzione}

Le sentenze della Corte incentrate sull'esecuzione pongono maggiormente l'accento sulla prevenzione di violazioni dei diritti umani simili in futuro. In tale ambito, la Corte indica quali sono i quadri normativi esistenti chiaramente inadeguati e che necessitano di modifiche o quali nuovi quadri vanno esplicitamente posti in essere. Tutti questi dibattiti si svolgono, in ogni caso, nei dispositivi. È quindi compito del Comitato dei Ministri e del Dipartimento per l'esecuzione delle sentenze identificare tali direzioni specifiche.

Il caso Cumpănă e Mazăre c. Romania, in cui la Corte ha evidenziato una regola quasi assurda secondo la quale le condanne penali per diffamazione non sono mai giustificabili ex art. 10 qualora una qualsiasi esternazione diffamatoria riguardi una materia di pubblico interesse, rappresenta un esempio importante in termini di modifiche legislative. ${ }^{53}$ Qui la Corte ha stabilito che.

Nonostante la condanna sia, di principio, una questione rientrante nelle competenze delle corti nazionali, la Corte ritiene che l'imposizione di una condanna detentiva per un'offesa mediatica sia compatibile con la libertà di espressione giornalistica come garantita dall'art. 10 della Convenzione soltanto in circostanze eccezionali, ovvero quando sono stati seriamente lesi diritti fondamentali come, per esempio, in casi di discorsi di odio o di incitamento alla violenza. ${ }^{54}$

Nel caso Belpietro c. Italia, la Corte ha riaffermato tale standard, evidenziando pertanto la necessità di modifiche specifiche nello scopo delle condanne detentive.

Negli anni recenti, la Corte ha sviluppato una serie di casi sulla libertà di stampa in cui la Corte collega la violazione dei diritti alla mancanza di tutele nazionali per la libertà di stampa. In confronto ai dispositivi che creano standard, ciò offre un approccio casistico molto più focalizzato sull'esecuzione. In questo modo, la Corte è in grado di trattare direttamente l'adeguatezza dei rimedi nazionali preesistenti e di offrire alle autorità na-

53 Cumpănă e Mazăre c. Romania, Ricorso n. 33348/93 (2004).

54 Ibid., paragrafo 115. 
zionali direttive specifiche al fine di creare un quadro normativo nuovo. Due esempi positivi di tale approccio si trovano nei casi Editorial Board of Pravoya Delo e Shetkel c. Ucraina (2011) e De Telegraaf c. Paesi Bassi (2012).

Nel primo caso, Pravoye Delo, un quotidiano ucraino, aveva pubblicato una lettera anonima comparsa su un sito internet che accusava alcuni ufficiali di essere coinvolti in attività criminali. Il giornale aveva poi menzionato la fonte delle informazioni e specificato che il contenuto della lettera non era stato verificato e che avrebbe potuto essere, di conseguenza, falso. Uno degli ufficiali citati nella lettera ha chiesto i danni per diffamazione, citando in giudizio il consiglio editoriale e il caporedattore del quotidiano. La legge ucraina esenta i giornalisti dalla responsabilità civile per aver citato materiali già pubblicati altrove in altri canali stampa. Nel presente caso, le corti nazionali hanno, tuttavia, ritenuto che, posto che la normativa esistente non regolava la pubblicazione su siti internet, il giornalista avrebbe non potuto prevedere che l'esenzione non si applica in questo caso. La Corte ha evidenziato che l'Ucraina necessitava di una nuova legge che assicurasse ai giornalisti ucraini che non fosse "richiesto di risarcire i danni per diffamazione qualora essi non abbiano divulgato le informazioni intenzionalmente, abbiano agito in buona fede e verificato le informazioni, o se le parti danneggiate non hanno fatto uso delle possibilità di risolvere il contenzioso prima di adire alla corte". ${ }^{55}$ In questo modo, la Corte ha indicato, nel proprio giudicato, non solo che era richiesta una misura generale in forma legislativa, ma ha altresì evidenziato gli ambiti che la futura normativa avrebbe dovuto includere.

Il caso De Telegraaf c. Paesi Bassi riguardava il diritto di proteggere le fonti giornalistiche. La Corte ha ritenuto che tale diritto vada salvaguardato tramite garanzie processuali sufficienti, inclusa la garanzia ex ante di revisione da parte di un giudice o di un altro corpo decisionale indipendente e imparziale, prima che la polizia o il pubblico ministero abbiano accesso alle informazioni dalle quali si potrebbe risalire alle fonti. Rivista la legge olandese, la Grande Camera ha concluso all'unanimità che:

la qualità della legge era carente in quanto non vi era alcuna procedura accompagnata da un'adeguata tutela legale per la società appellante al fine di consentire una valutazione indipendente, mentre l'interesse dell'investigazione penale ha ignorato il pubblico interesse consistente nella protezione delle fonti giornalistiche. ${ }^{56}$

55 Editorial Board of Pravoya Delo e Shtekel c. Ucraina (2011), paragrafo 65.

56 De Telegraaf c. Olanda (2012), paragrafo 100. 
Come nel caso ucraino, la Corte ha richiamato, pur conservando il modello delle sentenze declaratorie, la necessità di una legislazione specifica da mettere in campo quale tutela procedurale per le libertà giornalistiche.

\subsection{Misure individuali specifiche e urgenti}

Come precedentemente trattato, la Corte considera le proprie sentenze in primis uno spazio per svolgere funzioni interpretative. Come corollario di ciò, la Corte ordina raramente specifiche misure individuali nelle proprie sentenze. ${ }^{57}$ La Corte adotta misure individuali specifiche e urgenti quando ritiene che i giudicati sostanziali non possano consentire qualsiasi altra soluzione alternativa. Tale approccio è per natura limitato e dimostra la cautela della Corte nell'impegnarsi direttamente nell'esecuzione delle proprie sentenze.

Nel campo della libertà di stampa, le misure individuali sono state utilizzate in due occasioni e hanno ottenuto risposte celeri da parte degli Stati riceventi. Il primo caso riguardava l'ordinazione di misure individuali specifiche relative all'incarcerazione di un giornalista. Nel caso Fattullayev c. Azerbaigian del 2010, ${ }^{58}$ atteso che non sussistono mezzi alternativi a specifiche misure individuali ("no real choice test" 59 ), e asserito che l'interferenza nel diritto alla libertà di espressione dell'appellante per mezzo dell'incarcerazione non era giustificabile a norma dell'art. 10, paragrafo 2 della Convenzione, la Corte ha stabilito che:

tenendo presenti le particolari circostanze del caso e l'urgente necessità di porre termine alle violazioni ex art. 10 della Convenzione, la Corte ritiene che lo Stato convenuto debba, quale uno dei mezzi per adempiere il proprio obbligo ai sensi dell'art. 46 della Convenzione, assicurare l'immediato rilascio dell'appellante. ${ }^{60}$

Non vi è alcun dubbio sul fatto che il presente caso abbia inviato un segnale forte da parte di una Corte che di rado ordina misure individuali urgenti riguardanti la vulnerabilità dei giornalisti nei confronti dell'incarcerazio-

57 Maestri c. Italia [GC], N. 39748/98, paragrafo 47, ECHR 2004-I; Assanidze c. Georgia [GC], n. 71503/01, paragrafo198, ECHR 2004-II; e Ilaşcu ed altri c. Moldavia e Russia [GC], N. 48787/99, paragrafo 487, ECHR 2004-VII.

58 Fattullayev c. Azerbaigian n. 40984/07, Sentenza (merito ed equa riparazione), Corte europea dei diritti dell'uomo (prima sezione), 22 aprile 2010.

59 Cf. Assanidze c. Georgia [GC], N. 71503/01, paragrafo 202.

60 Fattullayev c. Azerbaigian, paragrafo 177. 
ne. Ciò rappresenta un vantaggio rispetto alla casistica precedente della Corte, nella quale la Corte era restia a collegare la vulnerabilità dei giornalisti nei confronti di altri diritti sostanziali sanciti dalla Convenzione. ${ }^{61} \mathrm{La}$ decisione di richiedere una misura individuale urgente è compatibile con le preoccupazioni più ampie espresse dalla Corte con l'effetto "dissuasivo" di pratiche detentive. Crea, inoltre, un precedente per la considerazione della Corte nei confronti della detenzione arbitraria di giornalisti in futuro. ${ }^{62}$

Seguendo il precedente creato dal caso Fattullayev, la Corte non ha applicato il proprio approccio rivolto all'attestazione della mancanza di misure alternative (il citato "no real choice test") ad altri casi nei quali la vulnerabilità dei giornalisti è in gioco a causa di dure misure governative. È un fatto ben risaputo, anche nella casistica della Corte, che, oltre all'incarcerazione, i giornalisti divengono spesso vittime di attacchi fisici in democrazie fragili o deboli, nelle quali i governi si preoccupano più di sopprimere esternazioni a loro sfavorevoli che di assumere misure positive per proteggere i giornalisti.

Un esempio indicativo di ciò è dato dal caso Najafli c. Azerbaigian del 2012. Esso riguarda un giornalista oggetto di un attacco perpetrato da ufficiali di polizia mentre questi stava riportando una manifestazione. Venne attaccato nonostante avesse ripetutamente fatto presente di essere un giornalista. La Corte ha ravvisato la violazione dell'art. 3 e ha altresì svolto un'importante considerazione sui diritti dei giornalisti legati all'art. 10. La Corte ha stabilito che "non si può contestare il fatto che il maltrattamento fisico di giornalisti da parte di agenti dello Stato mentre essi stanno adempiendo i loro obblighi professionali ostacoli seriamente il loro diritto di ricevere e diffondere informazioni". ${ }^{63}$ Inoltre, la Corte ha ritenuto che un periodo di tre mesi tra l'accaduto e l'avvio dei passi procedurali rilevanti configuri una violazione dell'obbligo, per lo Stato, di mettere in campo un'investigazione effettiva seguendo gli aspetti procedurali dell'art. 3 della Convenzione europea dei diritti dell'uomo. Nonostante il forte nesso tra il pestaggio del giornalista e l'importanza della sicurezza e delle libertà dei giornalisti per la società, tale sentenza venne comunque fornita in forma dichiarativa. Fu un'opportunità mancata per la Corte di stabilire quali misure individuali urgenti sarebbero necessarie in tali circostanze. Per esempio, la Corte avrebbe potuto richiedere un processo rapido per gli agenti

61 Vedi, per esempio, Kılıçc. Turchia paragrafi 84-87.

62 Özgür Gündem c. Turchia.

63 Najaflic. Azerbaigian, paragrafi 68. 
coinvolti nel pestaggio e le scuse ufficiali nei confronti del giornalista. Tali misure individuali avrebbero rafforzato la convinzione più implicita della Corte secondo la quale i crimini contro i giornalisti richiedono rimedi effettivi e il loro riconoscimento come tali.

Il secondo caso relativo alla libertà giornalistica in cui la Corte ha abbandonato il proprio approccio dichiarativo riguarda il caso Youth Initiative for Human Rights c. Serbia del 2013. Tale caso è importante da vari punti di vista in quanto la Corte ha considerato l'organizzazione non governativa un fornitore di informazioni preziose per il pubblico e le sue attività di richiesta di informazioni ai servizi segreti in merito alla sorveglianza dati elettronica una legittima richiesta di informazioni. Nel caso in questione, la Corte ha valutato la mancanza di alternative percorribili (" $n o$ real choice test") ai sensi dell'art. 46 e compiuto un passo in avanti nei propri rimedi giurisprudenziali per due aspetti chiave. In primis ha affermato che:

L'esecuzione più naturale del presente giudicato, la quale meglio corrisponderebbe al principio della restitutio ad integrum, sarebbe consistita nell'assicurare che i servizi segreti serbi fornissero all'appellante le informazioni richieste (ovvero il numero di persone soggette alla sorveglianza elettronica nel corso del 2005). ${ }^{64}$

In secondo luogo, la Corte ha chiesto al governo serbo di fornire le informazioni richieste entro tre mesi.

Il caso Youth Initiative for Human Rights dimostra la flessibilità della valutazione dell'assenza di alternative ("no real choice test") sviluppata dalla Corte. La valutazione può comportare la richiesta di rilasciare giornalisti così come il loro diritto di ricevere e di fornire informazioni.

\subsection{Le sentenze pilota e quasi-pilota}

Fino ad oggi la Corte non ha emesso alcuna sentenza pilota in riferimento alle libertà giornalistiche. Le sentenze pilota vennero originariamente sviluppate al fine di contrastare il problema del carico di lavoro della Corte analizzando i difetti sistemici e sistematici in un caso tra migliaia e di rinviare il caso all'ordinamento nazionale di provenienza fornendo una guida sulla risoluzione di predetti problemi (Leach et al. 2010). Uno sguardo generale sulle sentenze pilota fino ad ora dimostra che la Corte continua ad utilizzare tale procedura in modo pragmatico per risolvere un ampio nu-

64 Youth Initiative for Human Rights c. Serbia, paragrafo 32. 
mero di casi pendenti. ${ }^{65}$ I casi riguardanti la libertà giornalistica sono un'occorrenza regolare dinanzi alla Corte con ripetute violazioni dei diritti. Si potrebbe comunque argomentare che i casi riguardanti la libertà mediatica provenienti da determinati Paesi non sono mai così numerosi da legittimare l'uso della procedura delle sentenze pilota.

Al contrario, vediamo che la Corte ha fornito una serie di sentenze ai sensi dell'art. 46 che si riferiscono a misure generali nel campo delle libertà giornalistiche. Tali sentenze vengono spesso definite "sentenze quasi-pilota" in quanto anch'esse fanno riferimento a problemi sistemici o sistematici ed evidenziano modi per risolvere gli stessi. Si differenziano dalle sentenze pilota per due motivi: I) la Corte non termina di esaminare casi che configurino simili violazioni; e (II) non è previsto alcun termine. Si capisce che le sentenze emesse ex art. 46 hanno l'obiettivo di inviare segnali forti agli Stati e di coadiuvarli nella rapida esecuzione in riferimento ai problemi identificati nelle sentenze della Corte.

In riferimento all'utilizzo dell'art. 46 nei casi che riguardano la libertà giornalistica, la Corte non segue una valutazione ben specificata. Nel caso Ürper e altri c. Turchia del 2009, riguardante la sospensione del quotidiano, la Corte si è discostata dalla propria abituale impostazione decisionale per analizzare il modo in cui le violazioni hanno avuto luogo e ha indicato nella sezione della sentenza dedicata all'art. 46 che le violazioni dei diritti dei giornalisti $e x$ art. 10 "derivano da un problema che scaturisce dalla legislazione turca, ovvero la sezione 6(5) della legge n. 371”. La Corte proseguì indicando che "vari altri casi riguardanti il medesimo argomento pendono momentaneamente dinanzi alla Corte. Senza pregiudicare il merito di tali casi, i fatti sopra indicati rivelano che si tratta di un problema di natura sistemica". ${ }^{66} \mathrm{Di}$ conseguenza, la Corte ha richiesto alla Turchia di modificare la sezione 6(5) della legge n. $3713^{67}$ per assicurare che non si non si ripetano, in futuro, la sospensione della pubblicazione e della distribuzione di

65 Mentre il procedimento della sentenza pilota può essere adottato in riferimento a qualsiasi diritto protetto dalla Convenzione, la recente esperienza della Corte mostra che è necessario un numero significativo di cause pendenti davanti alla stessa per poter adottare questa proccedura. Per quanto riguarda la diveristà delle questioni oggetto delle sentenze pilota, Broniowski, Hutten-Czapska e MC ed altri (diritto di proprietà); Ümmühan Kaplan (eccessiva durata dei procedimenti giudiziari interni); Kuriç ed altri (perdita della cittadinanza in seguito allo smembramento dell'ex Jogoslavia); Ališiç ed altri (impossibilità di recuperare i "vecchi” risparmi in valuta estera nell'ex Jugoslavia) e Torreggiani (condizioni carcerarie inumane o degradanti).

66 Ürper ed altri c. Turchia (2009), paragrafo 51.

67 Ibid. 
interi periodici. ${ }^{68}$ L'approccio della Corte nell'utilizzo dell'art. 46 non è, nel presente caso, dissimile all'utilizzo della procedura delle sentenze pilota, in quanto la Corte fa riferimento specifico ai casi che attualmente pendono dinanzi a essa.

Nel caso Manole e altri c. Moldavia, un caso riguardante la carenza di imparzialità dell'emittente televisiva pubblica, la Corte ha seguito un approccio diverso ${ }^{69}$, evidenziando innanzitutto che:

Lo Stato deve, nella propria funzione di garante ultimo del pluralismo, assicurare tramite le proprie leggi e nella prassi che il pubblico abbia accesso tramite la televisione e la radio ad informazioni imparziali ed accurate e ad una serie di opinioni e commenti che riflettano inter alia le diverse opinioni politiche presenti nel Paese e che i giornalisti e gli altri professionisti che operano nel settore dei media audiovisivi non siano impossibilitati a fornire tali informazioni e commenti. ${ }^{70}$

Ha poi indicato che gli standard specifici per assicurare l'imparzialità sviluppati dal Comitato dei Ministri del Consiglio d'Europa ${ }^{71}$ debbano guidare le attività legislative in tale ambito. Si tratta di una sentenza importante dal punto di vista dei rimedi giurisprudenziali in quanto la Corte ha indirizzato uno Stato verso standard specifici sviluppati dal Comitato dei Ministri per attuare il proprio giudicato. Infine, nonostante lo abbia sottolineato nel dispositivo della sentenza, la Corte ha proseguito affrontando la necessità di un quadro normativo, tenendo altresì presenti gli standard esistenti separatamente ex art. 46 del giudicato.

68 La Corte seguì un approccio simile nel caso Gözel e Özer c. Turchia nel 2010, dichiarando che l'articolo 6(2) della legge 3713 non corrispondeva agli standard dell'art. 10 e che la sua compatibilità con la Convenzione dovesse essere assicurata al fine di prevenire violazioni analoghe.

69 Manole ed altri c. Moldavia.

70 Ibid., paragrafo 107.

71 Ibid., paragrafi 51-54. La Corte fa riferimento, in particolare, alla Risoluzione n. 1 dei Ministri degli Stati partecipanti alla quarta Conferenza ministeriale europea sulle politiche sui mass media (Praga, 7-8 dicembre 1994), sul futuro del servizio pubblico (1994); alla Raccomandazione n. R (96) 10 del Comitato dei Ministri sulla garanzia di indipendenza delle emittenti pubbliche (1996); alla Raccomandazione $\operatorname{Rec}(2000) 23$ del Comitato dei Ministri sull'indipendenza e le funzioni delle autorità regolatorie per il settore della radiodiffusione (2000); alla Dichiarazione del Comitato dei Ministri sulla garanzia di indipendenza del servizio pubblico di radiodiffusione negli Stati membri (2006). 
La Corte ha stabilito che:

nel presente caso la Corte ribadisce che ha ravvisato una violazione dell'art. 10 scaturita, inter alia, da una serie di lacune nel quadro legislativo di TRM. Considera che lo Stato convenuto è giuridicamente vincolato ai sensi dell'art. 46 e deve adottare il prima possibile misure generali per rimediare alla situazione che ha condotto alla violazione dell'art. 10. Alla luce delle lacune rilevate dalla Corte, tali misure generali dovrebbero includere una modifica legislativa per assicurare che il quadro normativo soddisfi le esigenze dell'art. 10 e che prenda in considerazione la Raccomandazione del Comitato dei Ministri n. R(96)10 e le raccomandazioni del sig. Jakubowicz. ${ }^{72}$

Gli approcci adottati nei confronti delle misure generali ex art. 46 sono, di conseguenza, diversi. Nella sentenza Ürper e altri, la Corte si occupò dei casi pendenti dinanzi a sé e utilizzò l'art. 46 come modo efficiente per comunicare con lo Stato in riferimento alla soluzione di casi ripetuti. L'uso dell'art. 46 nella sentenza Manole non è legato a casi ripetuti, ma a una preoccupazione più ampia al fine di consentire che l'emittente pubblica moldava diventi un'istituzione imparziale. In altre parole, l'importanza sistemica della questione incita la Corte a indirizzare una riforma legislativa esplicita nell'art. 46.

\section{LA RESPONSABILITÀ DELLE CORTI E DEI PARLAMENTI: QUALE RUOLO PER UNA REVISIONE SUSSIDIARIA PER LA PROTEZIONE DELLA LIBERTÀ MEDIATICA?}

Le nuove teorie sulla responsabilità delle corti e dei parlamenti introdotte dalla Corte in risposta alla richiesta di rispettare la sussidiarietà del sistema della Convenzione non si riferiscono direttamente ai rimedi giurisprudenziali (Spano 2014: 12). Comportano, in ogni modo, importanti effetti domino sui rimedi giurisprudenziali della Corte. La teoria delle corti e dei parlamenti responsabili si basa essenzialmente sull'idea che le istituzioni nazionali che prendono in considerazione in maniera adeguata i principi della Convenzione nelle loro deliberazioni debbano godere di un margine di apprezzamento. La Corte ha acconsentito all'uso della presente teoria

72 Ibid., paragrafo 117. 
nel campo della libertà di espressione, inclusa la libertà giornalistica. ${ }^{73} \mathrm{Co}-$ me stabilito dalla Corte nel caso Animal Defenders, "[la] qualità della revisione parlamentare e giudiziaria circa la necessità della misura è di particolare importanza ... inclusa l'adozione di un rilevante margine di apprezzamento" ${ }^{74}$ Tale visione generale suggerisce che, qualora la Corte sia soddisfatta della qualità dell'impegno profuso in questioni che attengono alle libertà giornalistiche da parte delle corti e dei parlamenti nazionali, essa potrebbe non esercitare una revisione severa in un caso singolo e, a propria volta, non tenderebbe a intravvedere necessariamente una violazione. Ciò è accaduto nel caso Animal Defenders in cui la Grande Camera ha ritenuto che il divieto del Regno Unito di fare campagne pubblicitarie politiche in televisione e in radio che impedì al ricorrente, un gruppo socialmente impegnato che si occupa della protezione di animali, di trasmettere i propri spot televisivi, non configurasse un'interferenza sproporzionata con il diritto alla libertà di espressione dell'appellante.

La qualità delle deliberazioni parlamentari solleva la questione dell'interpretazione, da parte della Corte, del concetto di "qualità". Nel caso Animal Defenders, per esempio, la Corte ha prestato la dovuta attenzione al fatto che l'argomento fosse stato dibattuto da una serie di comitati e che fosse sostenuto da un gruppo transpartitico. ${ }^{75}$ La Corte prese quindi in considerazione la qualità dei processi parlamentari in questioni relative alla libertà di espressione per decisioni in merito assegnando un margine di apprezzamento agli Stati. Ciò potrebbe indebolire i rimedi giurisprudenziali della Corte in quanto potrebbe rendere la Corte più aperta verso diversi approcci sulla protezione della libertà di espressione più che verso il proprio approccio rivolto alla creazione di standard in base all'art. 10, giustificato dal ruolo preponderante della libertà di espressione nella Convenzione. Questo sviluppo è pertanto contrario al ruolo più attivo assunto dalla Corte ordinando misure individuali urgenti per proteggere giornalisti ed emettendo sentenze in base all'art. 46.

In riferimento alla teoria delle corti responsabili, vediamo che la Corte ha sviluppato standard più severi, come stabilito dalla Corte nel caso Von Hannover c. Germania:

73 Per gli esempi più recenti sull'argomento in questione, vedi Erla Hlynsdottir (N. 3) c. Islanda, Ricorso n. 54145/10, 2 giugno 2015, paragrafo 59, e l'opinione concorde del giudice Sajo.

74 Animal Defenders International c. Regno Unito, Ricorso n. 48876/08, 22 aprile 2013, paragrafo 10.

75 Animal Defenders, paragrafo 114. 
Se l'equilibrio raggiunto dalle autorità giudicanti nazionali è insoddisfacente, in particolare in quanto l'importanza o l'ambito di uno dei diritti fondamentali in gioco non sono stati adeguatamente presi in considerazione, il margine di apprezzamento accordato alle decisioni delle corti nazionali sarà ristretto. In ogni caso, se la valutazione è avvenuta alla luce dei principi che risultano dalla giurisprudenza consolidata, la Corte richiederebbe forti ragioni per sostituire il proprio punto di vista a quello delle corti nazionali, le quali godono, di conseguenza, di un margine di apprezzamento più ampio. ${ }^{76}$

Il riferimento all'utilizzo della giurisprudenza consolidata della Corte come causa per il deferimento alle corti nazionali suggerisce che non vi sia alcuna tensione apparente tra la teoria delle corti responsabili e i rimedi giurisprudenziali sviluppati dalla Corte nel campo delle libertà giornalistiche. Nella propria opinione convergente nel caso Erla Hlynsdottir (n. 3), il giudice Sajo, ha comunque richiamato alla cautela in tale ambito, evidenziando che riferimenti espliciti alla "sussidiarietà" della Convenzione in casi legati alla libertà di espressione potrebbe causare fraintendimenti da parte degli Stati in relazione ai loro obblighi di dare efficacia alla libertà di espressione. ${ }^{77} \mathrm{Nel}$ caso Erla Hlynsdottir (n. 3), la Corte ha rilevato, tramite il modello delle sentenze dichiarative, una violazione delle libertà giornalistiche in quanto ha ritenuto che l'equilibrio tra il diritto al giusto processo e i diritti e gli obblighi di un giornalista di impartire informazioni non fossero stati colpiti. Significativamente, la Corte ha ritenuto che, riportando un processo penale in corso, "la buona fede di un giornalista andrebbe valutata sulla base della conoscenza e delle informazioni a lui o lei disponibili al tempo in cui venne scritto o vennero scritti l'articolo o gli articoli in questione". ${ }^{78}$

76 Von Hannover c. Germania (No. 2) [GC], n. 40660/08 e 60641/08, paragrafo 107, CEDU 2012.

77 Erla Hlynsdottir (n. 3) c. Islanda, Ricorso n. 54145/10, l'opinione concorrente del giudice Sajo.

78 Vedi Erla Hlynsdottir (n. 3) c. Isalnda, Ricorso n. 54145/10, 2 giugno 2015, paragrafo71. 


\section{VALUTARE I RIMEDI GIURISPRUDENZIALI DELLA CORTE E STRADE PER IL FUTURO}

Nella sezione precedente, ho esaminato i rimedi giurisprudenziali della Corte europea dei diritti dell'uomo nel campo delle libertà giornalistiche. L'analisi mostra che la Corte dispone di un ampio numero di strumenti a sua disposizioni per consolidare le libertà giornalistiche nei contesti nazionali. Oltre alla propria giurisprudenza tradizionale volta alla creazione di standard nel campo della libertà dei media, la Corte ha sviluppati massime improntate sull'esecuzione e misure generali urgenti che offrono protezione ai giornalisti e a coloro che eseguono funzioni giornalistiche, così come le sentenze $e x$ art. 46 che identificano nello specifico la legislazione nazionale quale causa ultima della mancanza di effettiva implementazione e che richiedono una modifica normativa. Nel complesso, il ruolo della Corte, recentemente identificato come "partner strategico" del Comitato dei Ministri nell'esecuzione delle sentenze e nella prevenzione di ripetute violazioni, ha trovato riflesso nel campo delle libertà e delle protezioni dei giornalisti.

Contro tale sfondo, il presente capitolo ha anche cercato di dimostrare che l'uso, da parte della Corte, della richiesta di misure generali urgenti o di misure generali specifiche è stato tuttalpiù sporadico e non ha, fino a ora, tenuto in considerazione la generale atmosfera deteriorante in uno specifico Paese in relazione alle libertà giornalistiche.

In primis, nonostante i molteplici casi in cui la sicurezza e l'incolumità dei giornalisti sono state affrontate dalla Corte in riferimento all'Azerbaigian, la Corte ha richiesto misure individuali urgenti soltanto in un caso. Sarebbe stato più appropriato, per la Corte, prestare attenzione al calendario delle cause ripetute, anche nell'ordinare misure individuali urgenti. Essendo l'imposizione di misure individuali urgenti uno dei segnali più forti che la Corte è in grado di inviare ai Paesi membri, potrebbe essere più appropriato, nei Paesi in cui non si trova in pericolo un unico giornalista, ma un'intera professione, per la Corte, tenere in considerazione misure individuali urgenti in tutti i casi simili che si ripetano. L'alternativa, ovvero lasciare la richiesta di misure individuali urgenti all'iniziativa dello Stato nei propri piani d'azione consultandosi con il Segretariato del Comitato dei Ministri, non è praticabile. Tale metodo è soggetto a ritardi e agli Stati non viene, al momento, richiesto di inviare i piani d'azione prima che siano passati sei mesi dalla sentenza finale (Corte europea dei diritti nell'uomo 2009). Nei casi ripetuti in cui sono in gioco la sicurezza e il maltrattamento di giornalisti, la Corte può svolgere un ruolo importante assicurando il ri- 
lascio dei giornalisti o l'investigazione effettiva dei reati commessi contro di loro.

In secondo luogo, la gamma di misure urgenti ordinate finora dalla Corte è limitata. Essendo la protezione dei giornalisti in quanto tali una preoccupazione centrale della Corte, essa potrebbe beneficiare, sulla base dei singoli casi, dall'uso di una serie più ampia di misure individuali. Si potrebbe sostenere che non sussiste alcuna necessità per la Corte di ordinare la revisione di processi o la cancellazione di precedenti penali, in quanto essi sono parte dei rimedi giurisprudenziali consolidati del Comitato dei Ministri. Col tempo, il Comitato dei Ministri potrebbe ampliare il proprio ambito di problematiche individuali. Considerando il numero di sentenze arretrate che pendono dinanzi al Comitato dei Ministri, e sensibilità politiche in riferimento a quale caso scegliere per domandare misure individuali specifiche, la Corte potrebbe beneficiare maggiormente dall'evidenziare la possibilità di tali misure nella parte operativa delle proprie sentenze. Infatti, nel caso Youth Initiative c. Serbia, la Corte ha mostrato che la restitutio ad integrum nel campo delle libertà giornalistiche potrebbe richiedere misure urgenti specifiche secondo il caso specifico. Nonostante la Corte non abbia ancora fatto ciò, potrebbe considerare, in casi in cui i giornalisti hanno subito torture o maltrattamenti, di chiedere agli Stati di offrire un sostegno psicologico immediato ai giornalisti. Sei mesi o più dopo la conclusione di un caso costituisce un arco di tempo troppo lungo. Vi sono esempi di ciò nella giurisprudenza della Corte interamericana per i diritti umani. ${ }^{79} \mathrm{La}$ Corte europea dei diritti dell'uomo consentirebbe, segnalando al Comitato dei Ministri la necessità di rimedi urgenti, allo stesso di seguire l'esempio della Corte in altri casi. Di nuovo, la Corte deve prestare la dovuta attenzione all'urgenza della misura individuale prima di decidere di emettere una sentenza dichiarativa.

Come abbiamo visto nei casi contro la Turchia e la Moldavia, non sussiste alcun impedimento per la Corte ai sensi dell'art. 46 della Convenzione che non le consenta di fare riferimento ad una misura generale ben definita, come la richiesta di modificare uno statuto o di creare un nuovo quadro normativo. Analogamente alla mia critica nei confronti dell'uso delle misure urgenti, l'approccio della Corte per coinvolgere le decisioni ex art. 46 di questo tipo non ha, in ogni modo, portato chiaramente fuori dal previ-

79 Corte interamericana dei diritti umani, Vélez Restrepo e famiglia c. Colombia, questioni preliminari, merito, riparazioni e costi, Sentenza del 3 settembre 2012, Serie C n. 248, paragrafo 317; Corte interamericana dei diritti umani, Manuel Cepeda-Vargas c. Colombia, Sentenza del 26 maggio 2010, Serie C n. 213 al paragrafo 265. 
sto. Le decisioni di utilizzare l'art. 46 hanno dipeso, finora, da due fattori: (I) un'indicazione che casi simili pendono dinanzi alla Corte in riferimento alla medesima materia e (II) la necessità di un quadro legislativo in cui vengono attuati gli standard del Consiglio d'Europa.

Il secondo fattore dimostra che la Corte è cauta nell'uso dell'art. 46 quando identifica la non-esistenza di buone leggi. Come visto nel caso $\mathrm{Ma}$ nole, la Corte era diligente nel fare affidamento agli standard del Comitato dei Ministri al fine di evidenziare l'ambito della legislazione necessaria per assicurare che le emittenti pubbliche siano imparziali e diano accesso a visioni pluralistiche. Tale approccio mostra l'importanza del Comitato dei Ministri nel creare, dal punto di vista politico, standard specifici nel campo della libertà giornalistica. In altre parole, la Corte indicherebbe che l'obbligo di creare un "ambiente favorevole" viene adempiuto nel migliore dei modi da parte degli organi politici della società. Una volta sviluppati tali standard, sarebbe più facile per la Corte inserirli nei propri rimedi giurisprudenziali.

Un secondo modo per fare un uso più efficiente dell'art. 46 consisterebbe nell'evidenziare, da parte della Corte, l'esistenza di pratiche sistematiche. Un buon esempio di ciò è l'uso di accuse processuali contro giornalisti che poi però vengono prosciolti. La casistica "standard-setting" della Corte ha ampiamente riconosciuto che, se un processo si conclude con un'assoluzione, ciò non significa che non vi fu alcuna interferenza nella libertà di stampa. Posto il diffuso coinvolgimento dei pubblici ministeri nella libertà di espressioni, in particolare negli ordinamenti con il maggiore numero di violazioni della libertà di espressione, la Corte potrebbe far bene ad affrontare tale tematica alla luce dell'art. 46 della sentenza.

La Corte non dispone di alcuna sentenza pilota nell'ambito delle libertà giornalistiche. Si deve sottolineare che la Corte non è legata da "centinaia" di casi in tale ambito. Nella sentenza Rumf c. Germania vi furono, per esempio, solo 55 casi riguardanti la mancanza di rimedi per l'eccessiva lunghezza dei processi amministrativi, ma ciò bastò perché la Corte avviasse la procedura per un caso pilota. Inoltre, la Corte non è obbligata ad adottare un approccio puramente pragmatico alla procedura per la sentenza pilota. Seguendo l'esempio Manole, nei casi in cui la Corte rileva problemi sistematici e strutturali, essa potrebbe comunque invocare la procedura per la sentenza pilota pur senza l'esistenza di una lunga lista di casi. L'utilizzo di tale rimedio confermerebbe l'importanza della libertà mediatica e invierebbe un segnale forte sia agli Stati che al Comitato dei Ministri. 


\section{CONCLUSIONE}

Nel presente capitolo ho sostenuto che la Corte europea dei diritti dell'uomo può svolgere un ruolo sia più strategico che più simbolico nel rafforzare la protezione a livello europeo delle libertà giornalistiche. Ciò può avvenire tramite un uso maggiormente strutturato dei rimedi giurisprudenziali.

Difendo l'uso dei rimedi giurisprudenziali per tre motivi:

In primis, per ragioni di celerità politica, il Comitato dei Ministri potrebbe, senza l'assistenza della Corte, non chiedere misure individuali non standardizzate, quali il rilascio di un giornalista dal carcere o rendere disponibili informazioni dei servizi segreti. Per misure cos̀̀ specifiche e raffinate, il Comitato dei Ministri abbisogna di una guida specifica da parte della Corte al fine di offrire una migliore protezione ai giornalisti dei cui casi si occupa.

In secundis, il Comitato dei Ministri non è in grado di decidere in merito a misure individuali urgenti. I piani di azione di agenti governativi non devono essere presentati presso il Segretariato del Comitato dei Ministri prima che siano trascorsi sei mesi dalla sentenza finale e sono possibili e permessi ritardi oltre i sei mesi. Il sistema del Comitato dei Ministri non è, inoltre, concepito per misure individuali urgenti. La Corte deve svolgere il proprio ruolo in tale contesto al fine di offrire maggiore protezione ai giornalisti.

In tertiis, il Comitato dei Minstri non dispone della visione piena dei casi ripetuti pendenti dinanzi alla Corte europea dei diritti dell'uomo in materia di libertà giornalistiche. Da questa prospettiva, la Corte è il luogo migliore per evidenziare i problemi sistemici e strutturali, come avvenuto nel caso Ürper e altri. Tale ampia visione sulle proprie cause di cui gode la Corte la rende un partner importante per il Comitato dei Ministri nel provvedere soluzioni effettivi ai problemi emergenti in materia di libertà di stampa nelle varie giurisdizioni. La Corte non deve, comunque, attendere che i casi diventino "centinaia" in tale campo. Inoltre, data l'importanza delle libertà giornalistiche per il sistema della Convenzione nel suo complesso, ritengo che non dovrebbe attendere e adottare un atteggiamento più proattivo in riferimento alla serie di casi presenti. Il divario tra i propri standard sostanziali e l'implementazione dei giudicati deve rimanere una preoccupazione per la Corte.

Il sistema europeo dei diritti umani si contraddistingue per le sue caratteristiche uniche in riferimento alla divisione dei poteri tra la Corte, il Comitato dei Ministri e l'Assemblea parlamentare del Consiglio d'Europa per la protezione dei diritti umani e l'enfasi sul rispetto dei tentativi compiuti 
in buona fede da parte dei sistemi giuridici nazionali per il rispetto della Convenzione per mezzo del principio di sussidiarietà. Sullo sfondo della compresenza di più soggetti, ogni parte in causa deve agire sfruttando il proprio massimo potenziale e cooperare con gli altri al fine di proteggere uno dei diritti più rilevanti della Convenzione: la libertà giornalistica.

È possibile e altrettanto necessario che la Corte assuma un ruolo più attivo e un approccio di principio nei confronti delle libertà giornalistiche quale problematica legata ai rimedi giurisprudenziali. La dichiarazione del Comitato dei Ministri sulla protezione del giornalismo e la sicurezza dei giornalisti e di altri attori mediatici del 30 aprile 2014 ed i propri sforzi continuati per fornire raccomandazioni in materia, come nel precedente del caso Manole, sortiranno certamente un effetto positivo per lo sviluppo dei rimedi giurisprudenziali in tale campo.

\section{BIBLIOGRAFIA}

Agnostiou D. (2013), The European Court of Human Rights: implementing Strasbourg's judgments on domestic policy, Edinburgh University Press, Edimburgo.

Assemblea parlamentare del Consiglio d'Europa (2000), Risoluzione 1226 (2000) sull'esecuzione delle sentenze della Corte europea dei diritti dell'uomo.

Brems E. and Lavrysen L. (2013), "Procedural justice in human rights adjudication: the European Court of Human Rights”, Human Rights Quarterly, 35, 1, pp. 176200.

Çalı B. (2013) "Domestic courts and the European Court of Human Rights: towards developing standards of weak international judicial review?”, Opinio Juris, gennaio 2013, disponibile su http://opiniojuris.org/2013/01/11/domestic-courts-a nd-theeuropean-court-of-human-rights-towards-developing-standards-of-weakint ernational-judicial-review/, consultato il 21 luglio 2015.

Çalı B. e Koch A. (2014), "Foxes guarding the foxes? Peer review of human rights judgments by the Committee of Ministers of the Council of Europe", Human Rights Law Review, 14, 2, pp. 301-325.

Chayes A. e Chayes A. H. (1998), The new sovereignty: compliance with international regulatory agreements, Harvard University Press.

Comitato dei Ministri del Consiglio d'Europa (2000), Raccomandazione n. R (2000) 2 del Comitato dei Ministri agli Stati membri sul riesame o la riapertura di talune cause a livello nazionale a seguito di sentenze della Corte europea dei diritti dell'uomo, 19 gennaio 2000.

Comitato dei Ministri del Consiglio d'Europa (2004), Raccomandazione Rec(2004)6 del Comitato dei Ministri agli Stati membri sul miglioramento dei ricorsi interni, 12 maggio 2004. 
Comitato dei Ministri del Consiglio d'Europa (2008), Raccomandazione CM/ $\operatorname{Rec}(2008) 2$ del Comitato dei Ministri agli Stati membri sulle misure efficaci da attuare a livello interno per la rapida esecuzione delle sentenze della Corte europea dei diritti dell'uomo, 6 febbraio 2008.

Comitato dei Ministri del Consiglio d'Europa (2010) "Dichiarazione di Interlaken”, Conferenza di alto livello sul futuro della Corte europea dei diritti dell'uomo, Interlaken, 19 febbraio 2010, disponibile su https://wcd.coe.int/ViewDoc.js p?id=1591969, consultato il 21 luglio 2015.

Comitato dei Ministri del Consiglio d'Europa (2011) "Dichiarazione di Izmir", Conferenza di alto livello sul futuro della Corte europea dei diritti dell'uomo, Izmir, 26-27 aprile 2011, disponibile su https://wcd.coe.int/ViewDoc.jsp?id=178 1937, consultato il 21 luglio 2015.

Comitato dei Ministri del Consiglio d'Europa (2012) "Dichiarazione di Brighton", Conferenza di alto livello sul futuro della Corte europea dei diritti dell'uomo, Brighton, 19-20 aprile 2012, disponibile su https:/wcd.coe.int/ViewDoc.jsp?id= 1934031, consultato il 21 luglio 2015.

Comitato dei Ministri del Consiglio d'Europa (2014), Dicharazione del Comitato dei Ministri agli Stati membri sulla protezione del giornalismo e sulla sicurezza dei giornalisti e di altri operatori mediatici, 30 aprile 2014, disponibile su https:/ /wcd.coe.int/ViewDoc. jsp?id=2188999, consultato il 21 luglio 2015.

Consiglio d'Europa (2013), Protocollo n. 15 alla Convenzione europea dei diritti dell'uomo, Consiglio d'Europa, 24 giugno 2013, disponibile su http://conventio ns.coe.int/Treaty/en/Treaties/Html/213.htm, consultato il 21 luglio 2015.

Corte europea dei diritti dell'uomo (2009), "Action plans - action reports: definitions and objectives", Memorandum redatto dal Dipertimento per l'esecuzione delle sentenze della Corte europea dei diritti dell'uomo, M/Inf/DH(2009)29rev del 3 giugno 2009, disponibile su https://wcd.coe.int/ ViewDoc.jsp?id=1450969 \&Site=CM, consultato il 22 luglio 2015.

Corte europea dei diritti dell'uomo (2014), Written presentation by the Registrar of the European Court of Human Rights on the reform of the Court, 3rd Meeting of the Committee of Experts on the Reform of the Court (DH-GDR), Strasburgo, 24 settembre 2014, GT-GDR-F(2014)015.

Corte europea dei diritti dell'uomo (2015a), "Violations by article and by state: 1959-2014", General statistics of the European Court of Human Rights, disponibile su: www.echr.coe.int/Documents/Stats_violation_1959_2014_ENG.pdf, consultato il 21 luglio 2015.

Corte europea dei diritti dell'uomo (2015b), "Pending applications allocated to a judicial formation”, 30 giugno 2015, disponibile su www.echr.coe.int/Documen ts/Stats_pending_month_2015_BIL.pdf, consultato il 21 luglio 2015.

Corte europea dei diritti dell'uomo (2015c), "The pilot judgment procedure”, information note issued by the Registrar of the European Court of Human Rights, disponibile su www.echr.coe.int/Documents/Pilot_judgment_procedure_ENG. pdf, consultato il 21 luglio 2015. 
Corte europea dei diritti dell'uomo (2015d), "Pilot judgments", disponibile su www.echr.coe.int/Documents/FS_Pilot_judgments_ENG.pdf, consultato il 21 luglio 2015.

Corte europea dei diritti dell'uomo (2016), Regolamento della Corte, disponibile su www.echr.coe.int/Documents/Rules_Court_ENG.pdf consultato il 4 agosto 2015.

Hillbrecht C. (2014), Domestic politics and international human rights tribunals: the problem of compliance, Cambridge University Press.

Leach P. (2013), "The principles which can be drawn from the case-law of the European Court of Human Rights relating to the protection and safety of journalists and journalism", $\operatorname{MCM}(2013) 012$ [CDMSI(2013)Misc3], available at www.coe.int/t/dghl/standardsetting/media/Belgrade2013/MCM(2013)012_en_L each_ProtectionofJournalists.pdf, consultato il 22 luglio 2015.

Leach P., Hardman H., Stephenson S. e Blitz B. K. (2010), Responding to systemic human rights violations: an analysis of pilot judgments of the European Court of Human Rights and their impact at national level, Intersentia, Antwerp/Oxford/Portland.

McGonagle T. (2014), "Report on the Round Table on safety of journalists: from commitment to action”, Council of Europe, Strasbourg, 19 May 2014, available at https://www.coe.int/t/dghl/standardsetting/media/CDMSI/Safety\%20of\%20Jo urnalists\%20report.pdf, consultato il 21 luglio 2015.

Mowbray A. R. (2004), The development of positive obligations under the European Convention on Human Rights by the European Court of Human Rights, Hart Publishing, Oxford and Portland, Oregon.

Shelton D. (2006), Remedies in International Human Rights Law, Oxford University Press, Oxford.

Spano R. (2014), "Universality or diversity of human rights? Strasbourg in the age of subsidiarity”, Human Rights Law Review, 14, 3, pp. 487-502. 